\title{
Standardization and quality evaluation of herbal drugs
}

\author{
Shulammithi $\mathrm{R}^{1}$, Sharanya $\mathrm{M}^{2}$, Tejaswini $\mathrm{R}^{3}$, Kiranmai $\mathrm{M}^{4} *$ \\ ${ }_{1,2,4}$ Department of Pharmaceutical Chemistry, Bharat Institute of Technology, Jawaharlal Nehru Technological \\ University, Telangana-501510. \\ ${ }^{3}$ Department of Pharmaceutical Chemistry, Bharat School of Pharmacy, Jawaharlal Nehru Technological \\ University, Telangana-501510.
}

\begin{abstract}
The term "herbal drugs" denotes plants or plant parts that have been converted into phytopharmaceuticals by means of simple processes involving harvesting, drying, and storage. A practical addition to the definition is also to include other crude products derived from plants, which no longer show any organic structure, such as essential oils, fatty oils, resins, and gums. There is increasing awareness and general acceptability of the use of herbal drugs in today's medical practice. Although, most of these applications are unorthodox, it is however a known fact that over $80 \%$ of the world population depends on herbal medicines and products for healthy living. This rise in the use of herbal product has also given rise to various forms of abuse and adulteration of the products leading to consumers and manufacturers disappointment and in some instances fatal consequences. The challenge is innumerable and enormous, making the global herbal market unsafe. Evaluation of herbal drug is an important tool in the formulation of high quality herbal products. This review seeks to enlighten stakeholders in herbal medicine on the need to establish quality parameters with the help of advanced analytical tools and well defined standardization methods in ensuring the safety of the global herbal market. The processes of good quality assurance and standardization of herbal medicines and products using various spectroscopic, chromatographic and electrophoretic methods were also discussed. In fact, the research field of quality control of herbal medicines is really an interdisciplinary research. It needs crossover of chemistry, pharmacology, medicine and even statistics to provide a platform for the quality control of traditional herbal medicines and further to discover the novel therapeutics composed of multiple chemical compounds.
\end{abstract}

Keywords: Herbal drugs, Standardization, Quality control, Interdisciplinary research, Chromatography, Electrophoresis, $H P-L C$ and $G C-M S$.

\section{Introduction}

Standardization of herbal formulations is essential in order to assess of quality drugs, based on the concentration of their active principle, physical, chemical, physcio-chemical standardization and in vitro, in-vivo parameters[1]. Natural products have been our single most successful source of medicines. Each plant is like factory capable of synthesizing unlimited no of highly complex and unusual chemical substance whose structure covered otherwise escape the imagination forever. It is necessary to maintain reproducible efficacy and safety of phyto pharmaceutical therefore if phytopharmaceutical have to regard as rational drug should be standardized and pharmaceutical quality must be approved.

WHO stresses the importance of the qualitative and quantitative methods for characterizing the samples, quantification of the bio-markers or chemical markers and the finger print profile. If a principle active component is known, it is most logical to quantify this compound. Where active ingredients contributing to therapeutic efficacy are known, botanical preparations should be standardized to their compounds; where the active ingredients are not yet known, a marker substance which should be specific for the botanical plants are chosen for analytical purpose [2,3].

\section{Herbal drugs:}

Herbal drugs are of two types:

$>$ Single/ crude drug

$>$ Multiple herbal formulations

\section{Single /crude drugs:}

$>$ All mainly whole, fragment or cut plant, plant parts usually dried forms, but sometimes fresh.

$>$ It also includes algae, fungi and lichen. 


\section{Multiple herbal formulations:}

Formulations are obtained by subjecting herbal ingredients to various manufacturing process such as extraction, distillation, expression, fractions, partition, chromatography and formulations.

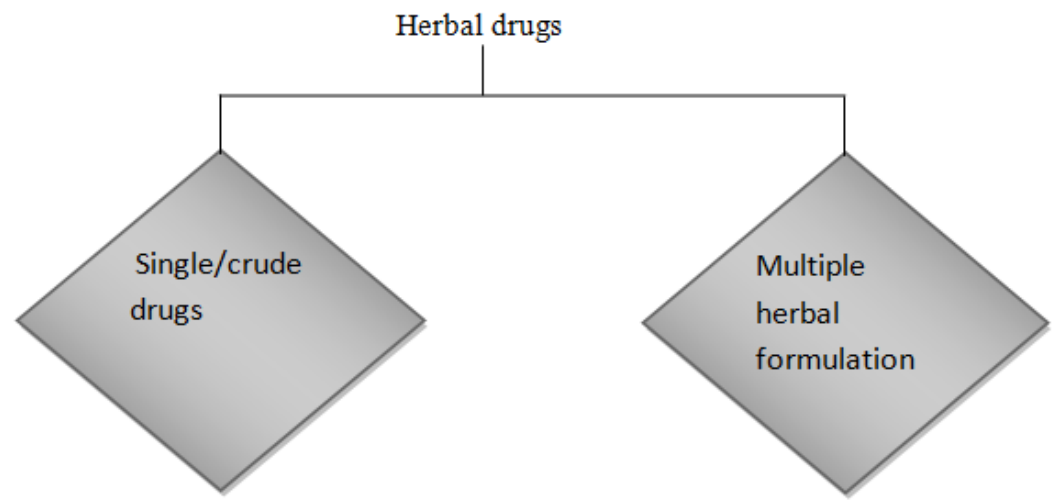

Fig.1 Multiple herbal formulations

\section{Quality Control}

It includes safety in herbal drugs, toxicity in herbals and their interactions

\section{Safety in herbal drugs:}

Major difference in the assessment of quality, safety and efficacy would hinder free circulation of herbal medicinal products may represent a risk for consumers. The complexity of herbal drug preparation and the interpretation of bibliographic data on safety and efficacy reflecting the experience gathered during longterm use are best addressed by involving specific expertise and experience. Safety and efficacy of complex biological products, such as herbal medicines products, are directly linked to pharmaceutical details such as the way of production and the specification of extracts. A consideration quality of herbal drugs may need more detailed information on aspects of agriculture products. The selection of seeds, condition of cultivation, and harvesting represent an important aspect in producing a reproducible quality of herbal drugs. Ongoing discussion on good agriculture practices [GAP] for medicinal plants should be monitored regularly $[4,5,6]$

\section{Toxicity in herbals and their interactions:}

A part from efficacy, FDA is also charged with determining the safety of drug products, and not all botanicals/herbals harmless. In this context the reference to the incidence of 1991 and 1992 in Brussels, Belgium, In which 30 women treated with a Chinese herbal slimming preparation died from renal failure caused by the presence of aristocholic acid in it can be taken into account. One of the herbs had been incorrectly identified as a non toxic. So the importance on controlling the correct identification of herbal preparations should be taken into account from very beginning. In addition to the problem of incorrect plant identification, some mixtures may be toxic, particularly if they are misused. Important should be given to continuous surveillance and of actively requesting information rather than just collecting reports and even this can be considered as national program [7].

\section{Analytical evaluation technique in herbal drugs:}

In general quality control is based on three important pharmacopoeias. It is obvious that the content is the most difficult one to assess, since in most herbal drugs the active constituents are unknown. Sometimes markers can be used which are, by definition , chemically defined constituents that are of interest for control purpose, independent of whether they have any therapeutic activity or not. To prove identity and purity, criteria such as type of preparation sensory properties, physical constants, adulteration, contaminants, moisture, ash content and solvent residues have to be checked. The correct identity of the cured herbal material, or the botanical quality, is of prime importance in establishing the quality control of herbal drugs.

\section{Organoleptic or macroscopic evaluation:}

Organic evaluation of drugs by means of organs of sense (skin, eye, tongue, nose, and ear) or microscopic evaluation which include evaluation of drugs by color, odor, taste, size, shape, and special feature, like touch, texture, etc. it is the technique of qualitative evaluation based on the study of morphological and sensory profile of whole drugs.

The fractured surfaces in cinchona, quillia, and cascara barks and quassia wood are important characteristics. Aromatic odor of umbelliferous fruits and sweet taste of liquorices are the examples of this type 
of evaluation where odor of drugs depends upon the type and quality of odorous principles (volatile oils) present.

\section{Microscopic evaluation:}

It involves detailed examination of the drugs and it can be used to identify the organized drugs by their known histological characters. It is mostly used for qualitative evaluation of organized crude drugs in entire and power forms with help of microscopic. Using microscope detecting various cellular tissues, trichomes, stomata, starch granules, calcium oxalate crystals and aleuronic grains are some of important parameters which play important role in identification of certain crude drugs [8-12].

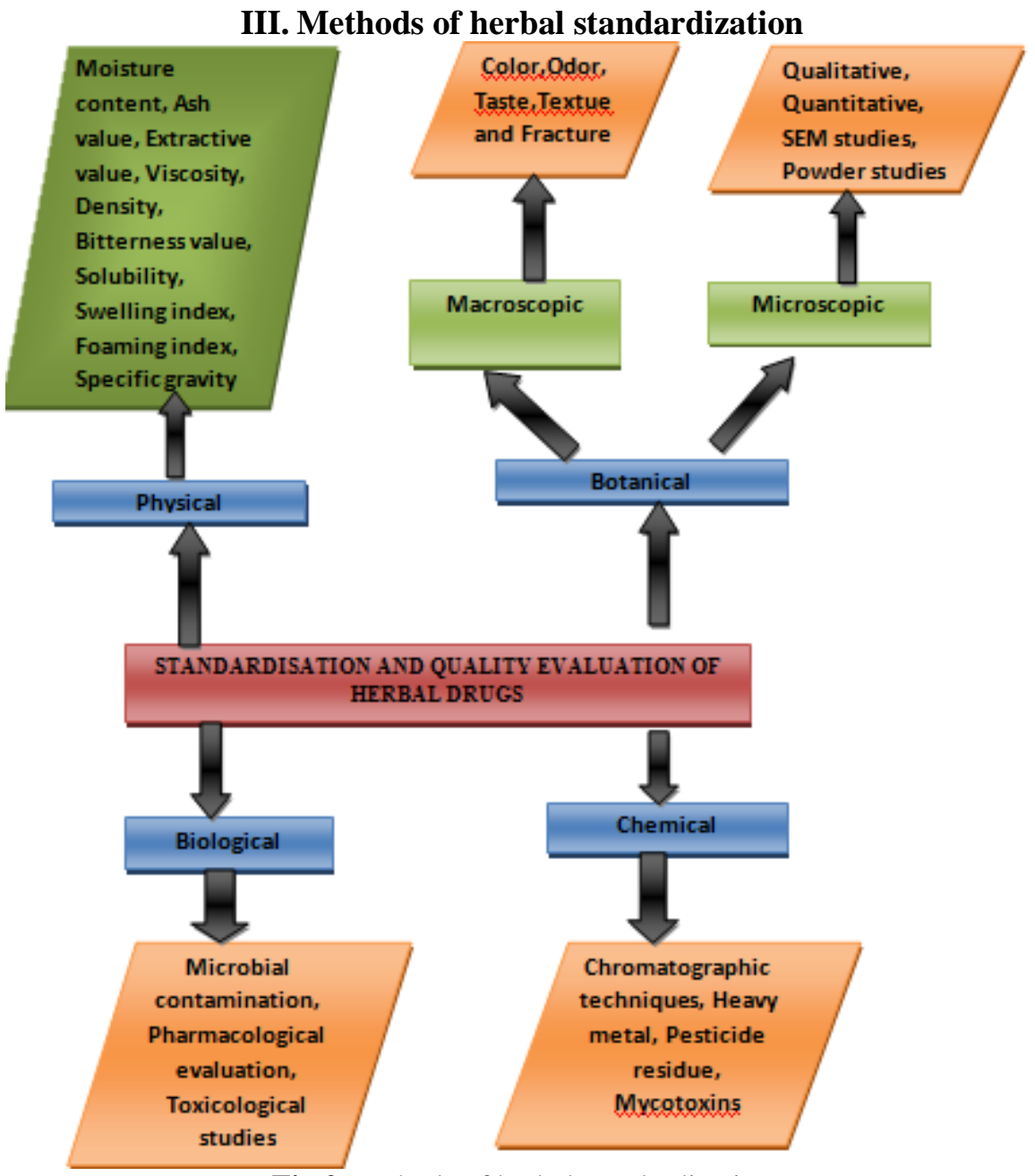

Fig.2 Methods of herbal standardization

Starch and hemicelluloses is identified by blue color with iodine solution, All lignified tissues give pink strain with phloroglucinol and $\mathrm{HCl}$ etc. mucilage is stained pink with ruthenium red can be used to distinguish cellular structure. Microscopic evaluation also includes study of constituents in the powdered drug by the use of chemical reagents.

Quantitative aspects of microscopy includes study of stomata number and index, palisade ratio, veinislet number, size of starch grains, length of fibers etc which plays a very important role in the identification of drug.

\section{Chemical evaluation:}

Most of drugs have definite chemical constituents to which their biological or pharmacological activity is attributed. Qualitative chemical test are used to identify certain drug or to test their purity. Isolation , purification, identification of active constituents is based on chemical methods of evaluation.

$>$ Evaluation test of resins : acid value, sulphated ash

$>$ Evaluation test of balsams: acid value, saponification value, bester values.

$>$ Evaluation test of volatile oils : acetyl and ester values 
$>$ The qualitative chemical tests are useful in identification of chemical constituents and detection of adulteration.

\section{Physical evaluation:}

Physical constants are sometimes taken into consideration to evaluate certain drugs. These include moisture content, specific gravity, optical rotation, refractive, melting point, viscosity and solubility in different solvents. All these physical properties are useful in identification and detecting of constituents present in plants.

\section{Biological evaluation:}

Some drugs have specific biological and pharmacological activity which is utilized for their evaluation. Actually this activity is due to specific type of constituents present in the plant extract. For evaluation the experiments were carried out on both intact and isolation organs of living animals. With the help of bioassays, strength of drug in its preparation can be evaluated [13-15].

\section{Chromatography techniques: \\ TLC (Thin layer chromatography):}

TLC was the most common, versatile methods of choice for herbal analysis before instrumental chromatography methods like gas chromatography and HPLC were established. Even now a day's TLC is still frequently used for the analysis of herbal medicines since various pharmacopeias such as Indian herbal pharmacopeia, Ayurvedic pharmacopeias, American herbal pharmacopeias, and Chinese drugs monographs. Rather TLC is used as an easier method of initial screening with a semi-qualitative evaluation together with other chromatography techniques as there is relative less change in the simple TLC separation of herbal medicines the with instrumental chromatography.

TLC is a technique in which solute undergoes distribution between two phases a stationary phase acting through adsorption and mobile phase in the form of liquid. The adsorbent is relatively thin, uniform layer of drug finely powdered material apply to glass, plastic, metal sheet/plate. Glass plates are the mostly commonly used. Separation may also be achieved on the basis of partition /a combination of partition and adsorptions depending upon the particular support its use with different solvent.

Identification can be effected by observation of spots of identical Rf value and equal magnitude obtained, respectively with an unknown and a reference sample chromatography on the sample plate. A visual comparison of the size and the intensity of spots usually serve for semi-quantitative estimation. TLC has advantages of many folded possibilities of detecting in analysis herbal medicines. In addition TLC is rather simple and can be employed for multiple sample analysis. For each plate more than 30 spots of sample can be studied. CA MAG video stored system and TLC QA-UV methods it's is possible to get useful qualitative and quantitative information from the developed TLC plates.

For example the four sample of cordyceps sinensis from that joint product of china and Japan cooperation has more valuable medicinal effect compared to other as they contain the most effective component "cordycepine" more over with the help of imagine analysis and digitized technique developed in computer science, evaluation of similarities between different samples is also possible.

TLC is being employed extensively for the following reasons:

$>$ It enables rapid analysis of herbal extracts with minimum sample clean-up requirement.

$>$ It provides qualitative and semi-quantitative information of the resolved compounds.

In TLC fingerprinting, the data that can be recorded using a high-performance TLC (HPTLC) scanner which includes information like chromatogram, retardation factor (Rf) values, the color of the separated bands, their absorption spectra, $\lambda$ max and shoulder inflection/s of all the resolved bands. All of these, together with the profiles on derivatization with different reagents, represent the TLC fingerprint profile of the sample. The information so generated has a potential application in the identification of an authentic drug, in excluding the adulterants and in maintaining the quality and consistency of the drug. TLC fingerprinting was done on the methanolic extract of Sitopaladi churna for determination of piperine using Silica Gel G plate and Toluene: Ethyl acetate: Formic acid (5:3.5:0.5 v/v/v) as mobile phase. Retention factor of piperine was found to be 0.69 (shown by peak 7) at $342 \mathrm{~nm}$. 


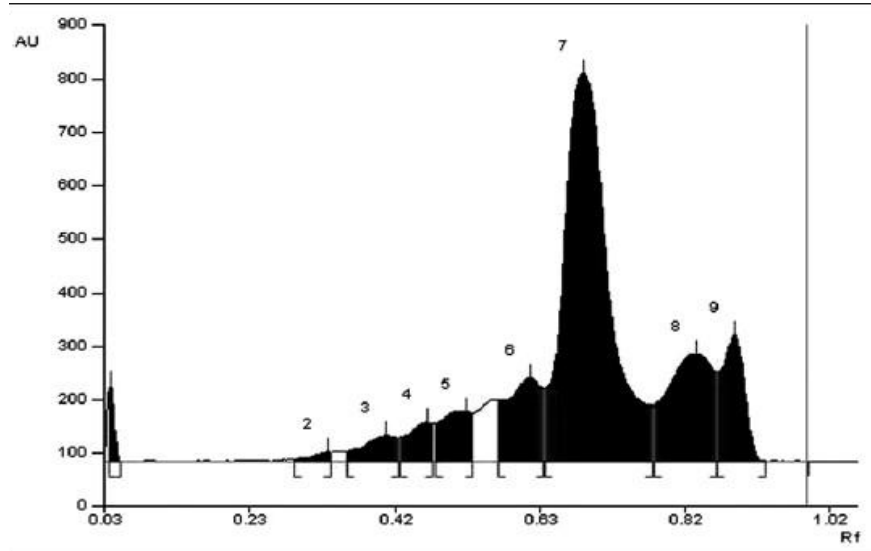

Fig.3 TLC of Sitopaladi churna

\section{Electrophoretic method:}

Capillary electrophoresis was introduced in early 1980s as a powerful analytical and separation technique and has been developed almost explosively. It allows an efficient way to document the purity/complexity of a sample and can handle virtually every kind of charged sample components ranging from simple inorganic ions to DNA. Thus, there was an obvious increase of electrophoretic methods, especially capillary electrophoresis, used in the analysis of herbal medicines in last decades. The more or less explosive development of capillary electrophoresis since its introduction has to a great extent paralleled that of liquid chromatography. Most of the used techniques are capillary zone electrophoresis (CZE), capillary gel electrophoresis (CGE) and capillary isoelectric focusing (CIEF). CE is promising for the separation and analysis of active ingredients in herbal medicines, since it needs only small amount of standard and can analyzed samples rapidly with good separation ability. Also, it is a good tool for producing the chemical finger prints of the herbal medicines, since it has similar technical characteristics of liquid chromatography. Recently, several studies dealing with herbal medicines have been reported and two kinds of medicinal compounds, i.e. alkaloids and flavonoids, have been studied extensively.

In general, $\mathrm{CE}$ is a versatile and powerful separation tool with high separation efficiency and selectivity when analyzing mixtures of low-molecular-mass components. However, the fast development in capillary electrophoresis causes improvement of resolution and throughout rather than reproducibility and absolute precision. On successful approach to improve the reproducibility of the both mobility and integral data has been on internal standards. But many papers were published unfortunately revealed the limited images on the real possibilities of CE in the field of finger print herbal medicines [16-24].

\section{Gas Chormatography:}

Gas chromatography also known as gas liquid chromatography, Its is a technique for separation of mixtures of mixtures into components by a process which depends on the redistribution of the components between a stationary phase or the support material in the form of a liquid, solid or combination of both and a gaseous mobile phase.

It is well-known that many pharmacologically active components in herbal medicines are volatile chemical compounds. Thus, the analysis of volatile compounds bys gas chromatography is very important in the analysis of herbal medicines. The GC analysis of the volatile oils has a number of advantages. Firstly, the GC of the volatile oil gives a reasonable "finger print" which can be used to identify the plant. The composition and relative concentration of the organic compounds in the volatile oil are the characteristic of the particular plant and the presence of impurities in the volatile oil can be readily detected. Secondly, the extraction of the volatile oil is relatively straight forward and can be standardized and the components can be readily identified using the GC-MS analysis. The relatively quantities of the components can be used to monitor or assess certain characteristics of the herbal medicines. Changes in composition of the volatile oil may also be used as indicators of oxidation, enzymatic changes or microbial fermentation. The advantages of GC clearly lie in its high sensitivity of detection for almost all the volatile chemical compounds. Thus, over the past decades, GC is a most popular and useful analytical tool in research field of herbal medicines. Especially, with the use of hyphenated GC-MS instrument, reliable information on the identity of the compounds is available as well. However, the most serious disadvantages of GC are that it is not convenient for its analysis of the samples of the polar and non volatile compounds. For this, it is necessary to use tedious sample work-up which may include derivatization. Therefore, the liquid chromatography becomes another necessary tool for us to apply the comprehensive analysis of herbal medicines [25-26]. 
The first fully automated on-line GC-IR system was developed by Scott et al. Each eluted solute was adsorbed in a cooled packed tube, and then thermally regenerated into an infrared vapor cell. Subsequent to the IR spectrum being obtained, a small sample of the vapor was drawn from the IR cell into a low-resolution mass spectrometer and the mass spectrum was also be taken [27, 28].

\section{Gas Chromatography (GC)}

As some of the bioactive constituents of herbal medicines are volatile, GC analysis can often be used for authentication and quality control. The high selectivity of capillary columns enables separation of many volatile compounds simultaneously within comparatively short times. However, the most serious disadvantage of GC is that this method is not convenient for the analysis of samples which are thermo labile and non-volatile. The identification and quantification of chemical constituents present in poly herbal oil formulation (Megni) was done by GC for determination of Eugenol using DB-5 fused silica capillary column and helium as a carrier gas. The retention time was found to be 8.63 min (Fig. 4).

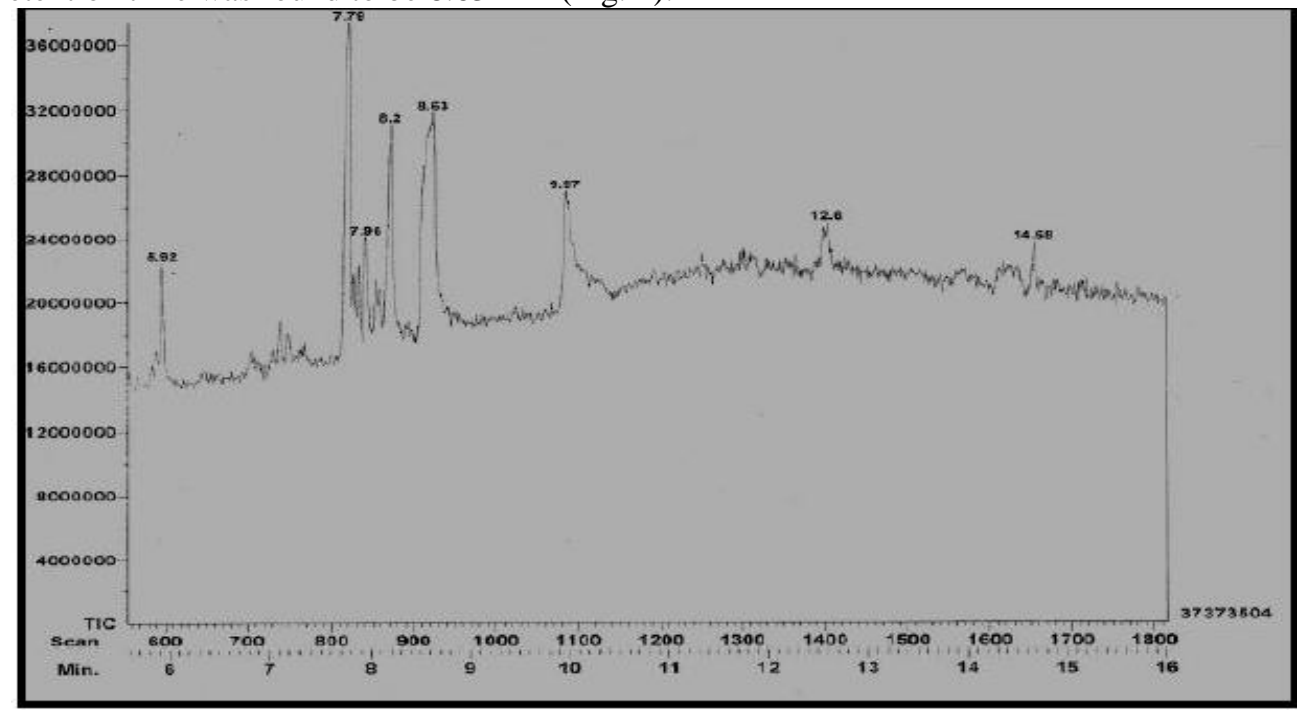

Fig.4 GC chromatogram of Eugenol $\left(\mathrm{R}_{\mathrm{t}}=8.63 \mathrm{~min}\right)$

High-Performance Liquid Chromatography (HPLC):

High performance liquid chromatography is also known as high pressure liquid chromatography in which the stationary phases consists of small particle $(3-50 \mu \mathrm{m})$ packing contained in a column with a small bore $(2-5 \mu \mathrm{m})$, one end of which is attached to a source of pressurized liquid eluent (mobile phase). The three forms of high performance liquid chromatography most often used are ion exchange, partition and adsorption. HPLC is a popular method for the analysis of herbal medicines. Because it is easy to learn and use and is not limited by the volatile or stability of the sample compound. In general, HPLC can be used to analyze almost all the compounds in the herbal medicines. Reversed-phase (RP) columns may be most popular columns used in the analytical separation of herbal medicines

It is necessary to notice that the optimal separation condition for the HPLC involves many factors, such as the different compositions of the mobile phase, their $\mathrm{pH}$ adjustment, pump pressures, etc. Thus, a good experiment design for the optimal separation seems in general necessary.

In order to obtain better separation, some new techniques have been recently developed in research field of liquid chromatography. These are micellar electro kinetic capillary chromatography (MECC), high speed counter current chromatography (HSCCC), low pressure size exclusion chromatography (SEC), reversedphase ion pairing HPLC (RPIPC-HPLC) and strong anion exchange HPLC (SAX-HPLC). They will provide new opportunities for good separation for some specific extracts of some herbal medicines. On the other hand, the advantages of HPLC lie on its versatility for the analysis of the chemical compounds in herbal medicines, however. The commonly used detector in HPLC, say single wavelength UV detector, seems to be unable to fulfill the task, since lots of chemical compounds in herbal medicines are non chromophoric compounds. Consequently, a mark increases in the use of HPLC analysis couples with evaporative light scattering detection (ELSD) in a recent decade demonstrated that ELSD is an excellent detection method for the analysis of nonchromophoric compounds.

This new detector provides a possibility for the direct HPLC analysis of many pharmacologically active components in herbal medicines, since the response of ELDS depends only on the size, shape, and number of the finger prints of the herbal medicines. Moreover, the qualitative analysis or structure elucidation of the chemical components in herbal drugs by simple HPLC is not possible, as they rely on the application of 
techniques using hyphenated HPLC, such as HPLC-IR, HPLC-MS, HPLC-NMR, for the analysis of herbal medicines.

\section{HPLC-DAD:}

It has become a common technique in most analytical laboratories in the world now. With the additional UV spectral information, the qualitative analysis of complex samples in herbal medicines turns out to be much easier than before. For instance, checking peak purity and comparing with the available standard spectrum of the known compound to the one in the investigated sample. Especially, with the introduction of electrospray mass spectrometry, the coupling of liquid chromatography and mass spectrometry has opened the new way to widely and routinely applied to the analysis of herbal medicines. HPLC chromatography finger prints can be the applied for documentation of complete herbal extracts with more information and on-line qualitative analysis becomes possible.

In last decades, the increasing usage of LC-MS and HPLC-DAD in the analysis of herbal medicines is quite obvious. Several good reviews have been published for the analysis of the bioactive chemical compounds in plants and medicines, in which the technique used most in HPLC, especially the hyphenated HPLC technique. Moreover, combined HPLC-DAD-MS technique take advantage of chromatography as a separation method and both DAD and MS as an identification method. DAD and MS can provide on-line UV and MS information for each individual peak in a chromatography. With the help of this hyphenation, in most cases, one could identify the chromatography peaks directly online by compression with literature data. Recently, the hyphenation between HPLC and NMR also available, which might become a vital and an attractive analytical tool for the analysis of herbal medicines. In fact the tendency of the hyphenation or multi-hyphenation of the chromatography with the common used four spectroscopic detectors.

Kankasava is a polyherbal formulation prepared with Kanaka and other ingredients which is used in chronic bronchitis, asthmatic cough and breathlessness. A simple, precise, accurate RP- HPLC method was developed for the quantitative estimation of atropine using column RP C-18 $(250 \mathrm{~mm} \times 4.6 \mathrm{~mm} \times 5 \mathrm{micron})$ and mobile phase which is mixture of methanol and $10 \mathrm{mM}$ dihydrogen phosphate buffer in a ratio of 50:50 v/v at a flow rate of $1 \mathrm{ml} / \mathrm{min}$, and analysis was screened with UV detector at $254 \mathrm{~nm}$. The retention time for standard atropine sulphate was found to be 4.0667 minutes (Fig. 5)[29-34].

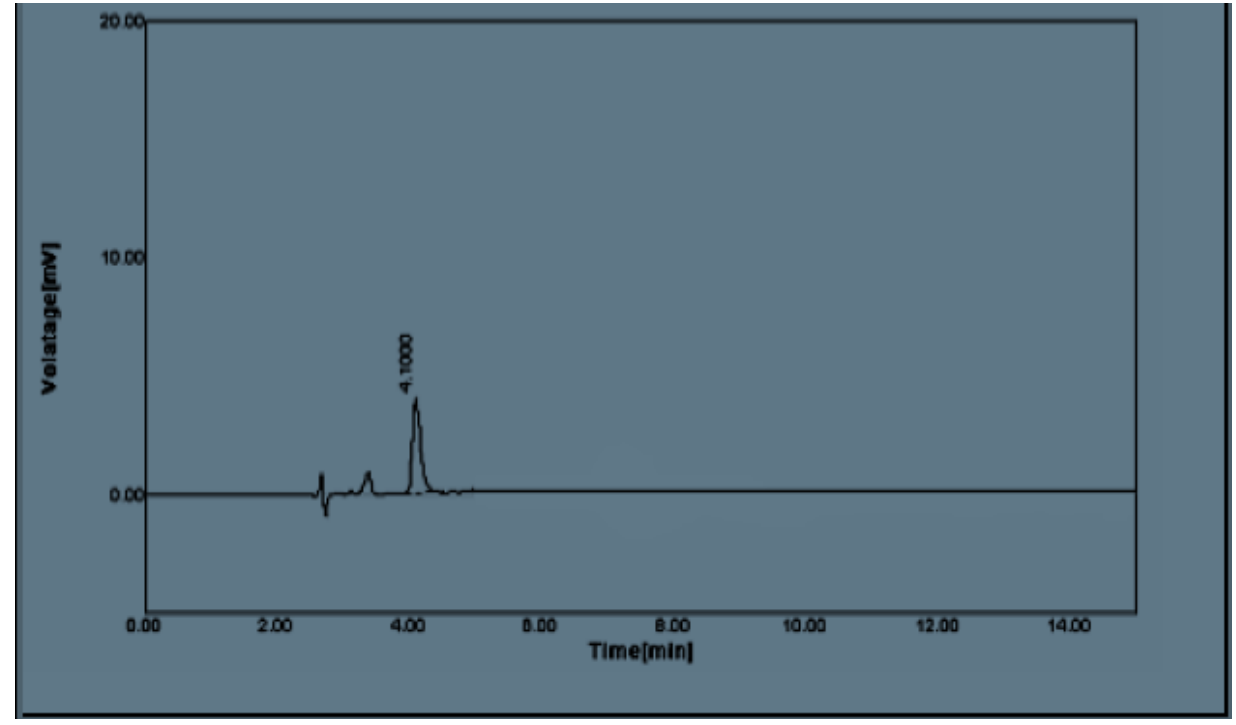

Fig.5 HPLC chromatogram for atropine sulphate $\left(\mathrm{R}_{\mathrm{t}}=4.0667 \mathrm{~min}\right)$

\section{Chromatographic fingerprinting:}

Chromatographic fingerprinting is the most powerful approach for the quality control of herbal medicines. Chromatographic fingerprint of Herbal Medicine is a chromatographic pattern produced from extract of some common chemical components which may be pharmacologically active or have some chemical characteristics. This chromatographic profile should be featured by the fundamental attributions of - integrity and -fuzziness or - sameness and - differences so as to chemically represent the herbal medicines investigated. This suggest that chromatographic fingerprint can successfully demonstrate both — sameness and - differences between various samples and the authentication and identification of herbal medicines can be accurately conducted even if the number and/or concentration of chemically characteristic constituents are not very similar in different samples of herbal medicine. Thus chromatographic fingerprint should be considered to evaluate the 
quality of herbal medicines globally; considering multiple constituents present in the herbal medicines [35-36]. This technique can be employed for identification and authentication as well as for determination of various adulterants and contaminants and for standardization purpose. In contrast to macroscopic, microscopic and other molecular biological methods this technique is not restricted to raw herbs, but can also be applied to pharmaceutical preparations. Chromatographic fingerprinting can be carried out using techniques such as thin layer chromatography (TLC), High performance thin layer chromatography (HPTLC), High performance liquid chromatography (HPLC), Gas chromatography (GC) and other hyphenated techniques.

\section{DNA fingerprinting:}

DNA analysis has been proved as an important tool in herbal drug standardization which is useful for the identification of phytochemically indistinguishable genuine drug from substituted or adulterated drug. DNA fingerprint genome remains the same irrespective of the plant part used while the phytochemical constituents will vary with the part of plant used, physiology and environment. The other useful application of DNA fingerprinting is the availability of intact genomic DNA specificity in commercial herbal drugs which helps in distinguishing adulterants even in processed samples.

\section{Types of DNA fingerprinting techniques used in plant genome analysis:}

Various types of DNA-based molecular techniques are utilized to evaluate DNA polymorphism which includes hybridization-based methods, polymerase chain reaction (PCR)-based methods and sequencing-based methods $[37,38]$.

\section{Hybridization-based methods:}

Hybridization based methods use cloned DNA elements or synthetic oligonucleotides as probes to hybridize the DNA of interest. The probes are labeled with radioisotopes or with conjugated enzymes which catalyze a color reaction to detect hybridization; Hybridization based methods include following steps:

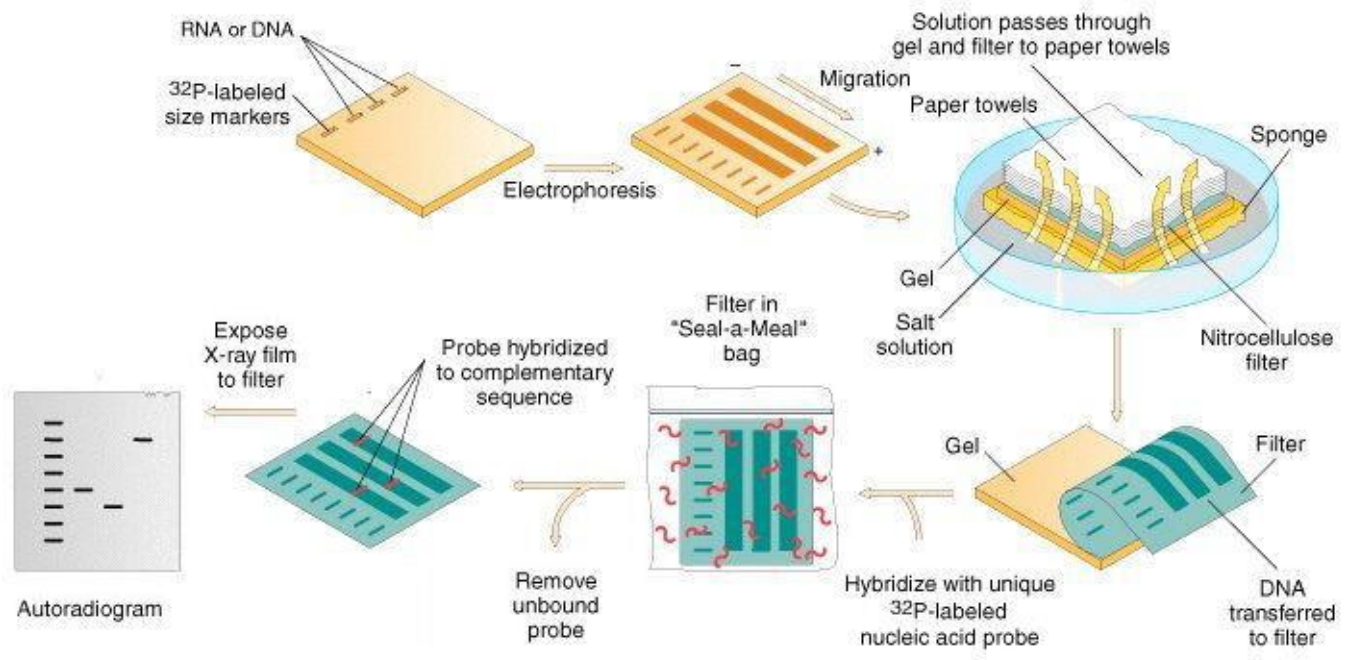

Fig.6 Hybridization based method

DNA is first extracted from plant cell by various methods of extraction such as Cetyl triethyl ammonium bromide (CTAB) method, Phenol/ Chloroform extraction method, DNA trap method etc. DNA is then cleaved with restriction enzyme after which it is subjected to gel electrophoresis. As DNA molecules have net negative charge, at neutral $\mathrm{PH}$, these molecules migrate towards the positive terminal when placed in an electric field in a process known as electrophoresis which is performed in an agarose or polyacrylamide gel. Nucleic acids are loaded into slots in the gel and allowed to migrate towards the positive terminal. The pores in the gel act to sieve the molecules. So that mobility of a particular nucleic acid species depends on its length. All the molecules of a particular size move at approximately the same rate through the gel, forming a band, which gradually increases in width during electrophoresis because of diffusion. After gel electrophoresis, DNA sequence is determined by Southern blotting technique. DNA molecules separated by gel electrophoresis are transferred to nitrocellulose or nylon membrane (Fig.6).

The DNA is denatured either prior to or during transfer by placing the gel in an alkaline solution, after which it is immobilized on the membrane by drying or UV induced cross linking to the filter. A radioactive DNA known as probe which are of two types (Tandem repeats which occurs as clusters among chromosomes and Dispersed repeats which are scattered all over chromosomes) containing the sequence of interest are then 
hybridized or annealed with the immobilized DNA on the membrane. The probe will anneal to form a double helix only with complementary DNA molecule on the membrane. Non annealed probe is then washed off the membrane, and the washed membrane is exposed to X-ray film that detects the presence of the radioactivity in the bound probe, after which autoradiogram is developed where the dark bands show the positions of DNA sequences that has hybridized with the probe. Hybridization-based methods include

a) Restriction Fragment Length Polymorphism (RFLP)

b) Variable Number Tandem Repeats (VNTR)

Restriction Fragment Length Polymorphism (RFLP) In this technique, plants may be differentiated by analysis of patterns derived from cleavage of their DNA. Restriction polymorphism is detected by using a hybridization probe. RFLPs involves fragmenting a sample of DNA by a restriction enzyme, which can recognize and cut DNA wherever a specific short sequence ( 4-6 base pair recognition site) occurs, in a process known as a restriction digest.. Hybridization of the membrane to a labeled DNA probe then determines the length of the fragments which are complementary to the probe. The resulting DNA fragments are then separated by length through a process known as agarose gel electrophoresis, and transferred to a membrane via the Southern blot procedure. PCR amplification of DNA is not required for this method.

\section{Limitations:}

$>$ Low sensitivity.

$>$ Requirement of large amount of high quality genomic DNA which is difficult to obtain from processed herbs.

> Stability and reproducibility are low because the quality of the assay highly depends on DNA quality and technical factors.

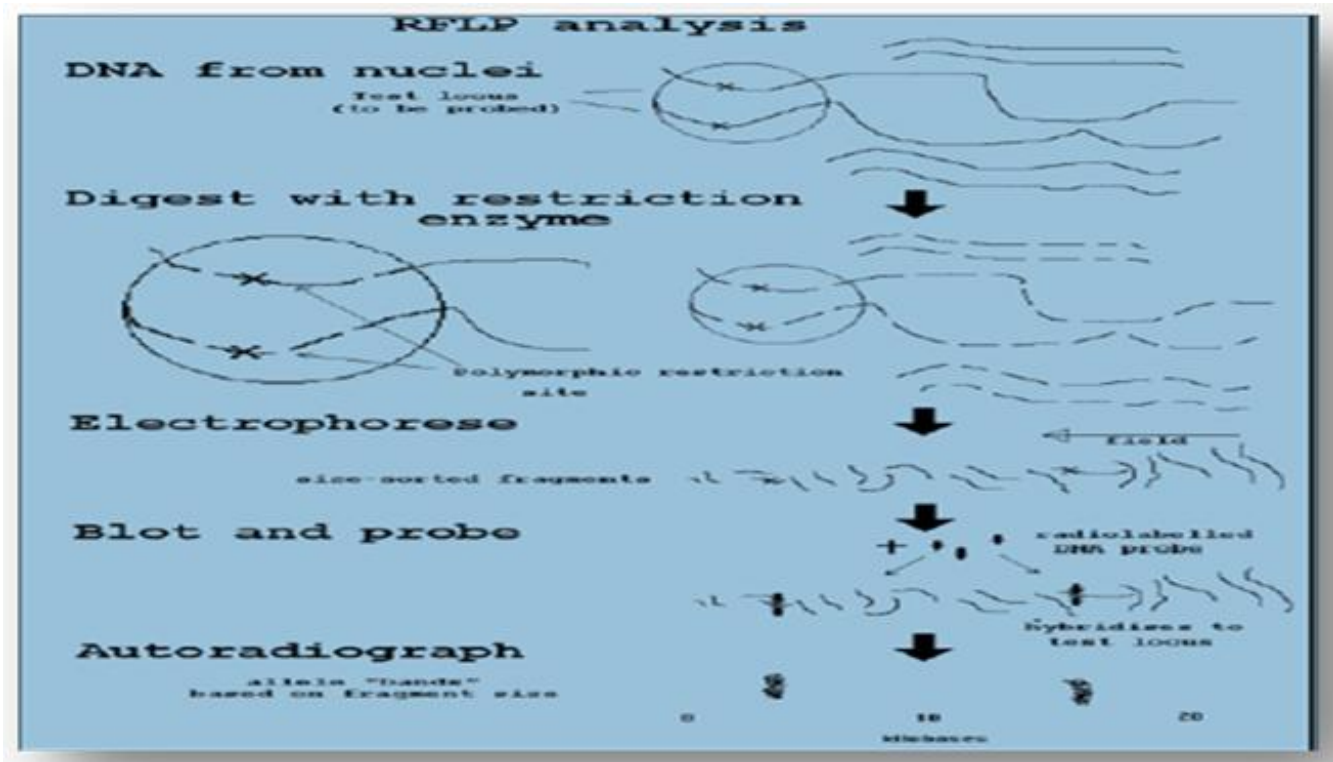

Fig.7 Steps involved in Restriction fragment length polymorphism

\section{Variable Number Tandem Repeats (VNTR):}

This technique is similar to RFLP only the probe used for southern blotting exists as tandem repeats which occur as cluster among chromosomes. They show variations in length between individuals and each variant acts as an inherited allele.

\section{Polymerase chain reaction based methods:}

PCR enzymatically multiplies particular DNA sequence or loci of a template DNA with the help of arbitrary or specific oligonucleotide primers. PCR based procedures are difficult to standardize due to use of different DNA polymerase, different buffer formulations and different equipments used.PCR based method involve following steps: Primers, original DNA (extracted from the plant cell) which is to be amplified, a specific type of DNA polymerase, and the necessary chemicals for DNA synthesis are mixed. Then following steps are carried out. 


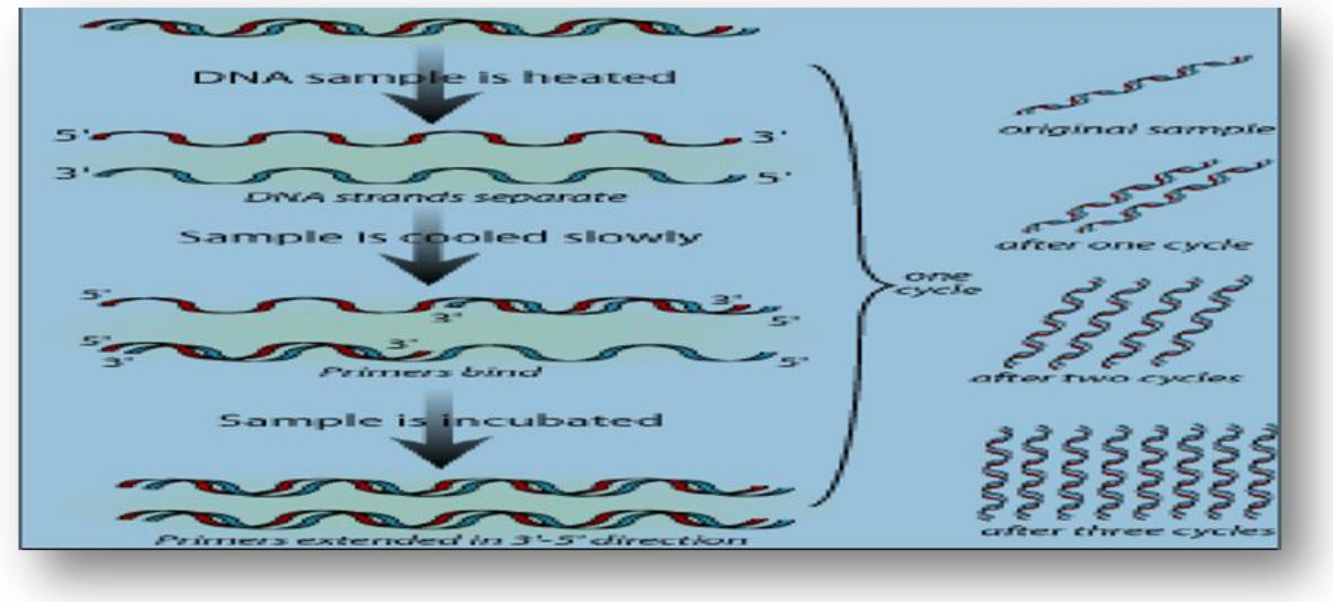

Fig.8 Steps involved in polymerase chain reaction (PCR)

$>$ Denaturation: DNA fragments are heated at high temperature (950C for 30 seconds or $970 \mathrm{C}$ for 15 seconds) which reduces DNA double helix to single helix to single strand which become accessible to primer.

$>$ Annealing: Here the temperature is lowered until the primers can hybridize, or bind to complementary regions on the DNA.

$>$ Extension: The primers are used by DNA polymerase to initiate synthesis and new complementary strand of DNA are made. Fig. 8.

The enzyme read opposing strand sequence and extends the primer by adding nucleotide in order in which they can pair Once this synthesis is finished, the steps are repeated i.e. denaturation, renaturation, and synthesis. However problems can occur when the plant material of interest contains compounds such as phenolics which can interact with DNA and directly inhibit DNA polymerase or damage the structural integrity of DNA. Various PCR based techniques include:

a) Randomly Amplified Polymorphic DNA (RAPD)

b) Arbitrarily Primed Polymerase Chain Reaction (AP-PCR)

c) Amplified Fragment Length Polymorphism (AFLP)

d) Simple Sequence Repeats (SSR)

Randomly Amplified Polymorphic DNA ( RAPD) and Arbitrarily Primed Polymerase Chain Reaction (AP- PCR) These methods are most commonly used for primary assay which helps in screening the differences in DNA sequence of two species of plants. In this method single arbitrarily chosen oligonucleotide is used as both the forward and reverse primer in PCR reaction. This sequence consists of about 10 nucleotide in case of RAPD and about 20 nucleotides in case of AP-PCR. Product is produced when the primer binds on opposite strands, in the reverse orientation and within an amplifiable distance. PCR fragments are generated from different locations of the genome, because there are multiple sites within the genome for the primer to bind. Thus, multiple loci may be examined simultaneously. Use of series of different primers, shows the generation of genetic fingerprint (Fig. 9).

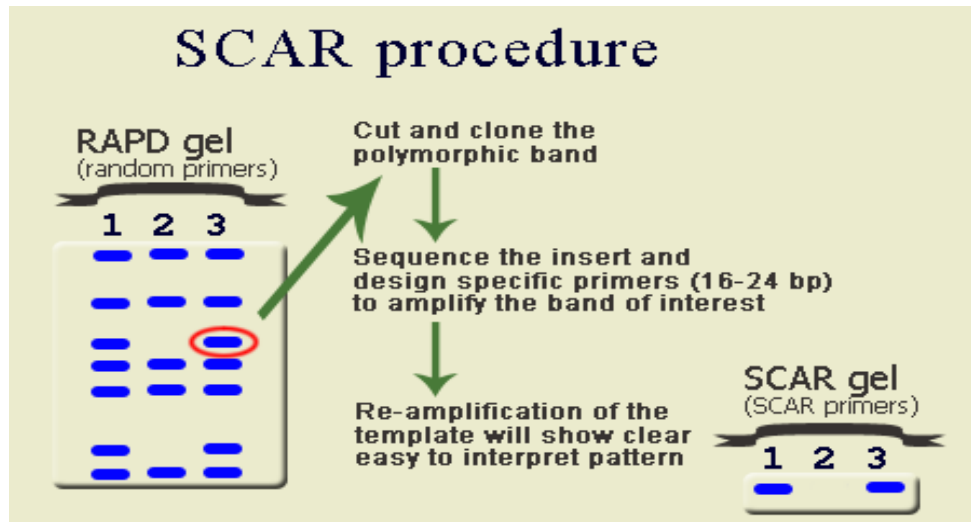

Fig. 9 Steps involved in RAPD 
Advantages:

$>$ High number of fragments.

$>$ Arbitrary primers are easily purchased, with no need for initial genetic or genomic information.

$>$ Only tiny quantities of target DNA are required.

$>$ Unit costs per assay are low.

$>$ Faster analysis.

$>$ Easy method.

Disadvantage:

$>$ Reproducibility between laboratories is low because the procedure is very sensitive to different PCR parameters especially to slight variation in annealing temperature due to low annealing temperatures required.

$>$ Highly sensitive to laboratory changes [39-40].

\section{Conclusion}

Plant materials are used throughout the developed and developing world as home remedies, in overthe-counter drug products, and as raw material for the pharmaceutical industry, and they represent a substantial proportion of the global drug market. The quality of herbal drugs is the sum of all factors which contribute directly or indirectly to the safety, effectiveness and acceptability of the product. Now a day the field of herbal drugs and formulation is very fast and there is still lot to explore on the subject of standardization of these. So, while developing an herbal formulation it is must to have all the related knowledge of that particular drug including all its organoleptic characters to phytoconstituents to pharmacological action to its standardization in respect to various parameters via various techniques. The problems of quality assurance of herbal medicines have been solved to great extent with the help of chromatographic DNA fingerprint analysis.

\section{References}

[1]. Satheesh madavi NN, kumud upadya, asha bishti, phytochemical screening and standardization of poly herbal formulation for dyslipisemia. Indian journal of physiology and pharmacology. 2011.

[2]. WHO. 1988. Quality Control Methods for Medicinal Plant Materials. World Health organisation,Genava.

[3]. WHO. 1992. Quality Control Methods for Medicinal Plant Materials. World organisation,Genava.

[4]. Eisenberg DM, kesseler RC, Foster C, norlock FE, Calkins DR, Delbanco TL. Unconventional medicine in the United States. Prevalence, costs and patterns of use. 1993

[5]. Blumenthal M, Brusse WR, Goldberg A, Gruenwald J, Hall T, Riggins CW, Rister RS, The complete german commission E monographs. Therapeutic guide to herbal medicines, the American botanical counsil, Austin, TX. 1998.

[6]. Brain KR and Turner TD. Practical Evaluation of phytopharmaceuticals. Wright Scientechnica Bristol. 1975.

[7]. Fahishi, A (1996) Complementary medicine. Vol.1. London: financial times amd health care publishing.Future system for the free movement of medicinal products in the European community.the rules governing medicinal products in the European community volume3; guidelines on the quality safety and efficacy of medicinal products for human use. 1989

[8]. Gupta MK and Sharma PK. Test Book of Pharmacognosy, Ayurvedic formulations, Pragati Prakashan Meerut Vol II, Ist edition.2007.

[9]. Herbone JB..Phytochemical methods, Chapman and Hall, London, New York, $2^{\text {nd }}$ Edition. 1928.

[10]. Kokate CK, Gokhale SB. Pharmacognosy. Nirali prakashan, Delhi. 2004.

[11]. Kokate CK, Purohit AP, Gokhale SB. Pharmacognosy, 31st edition Nirali Prakshan, 2005, 97-131.

[12]. Ansari SH, .Essentials of pharmacognosy, birla publications pvt 1td, 2001, 10-16.

[13]. Ahirwal B, Ahirwal D and Ram A.Evaluation of standards and quality control parameters of herbal drugs, souvenir, recent trends in herbal therapy. 2006; 25-2.9

[14]. Williamson E, Okpako DT, Evans F J. Pharmacological Methods in Phytotherapy Research, , Preparation and Pharmacological Evaluation of Plant Material.John Wiley and Sons, Chichester. 1996, 1(1).

[15]. Li N, Lin G, Kwan YW, Min ZD. Simultaneous quantification of five major biologically active ingredients of saffron by highperformance liquid chromatography, J. Chromatogr. A, 1999, 849(2), 349-355.

[16]. Li X N, Cui H, Song Y Q, Liang Y Z, Chau F T. 2003. Analysis of volatile fractions of Schisandra chinensis (Turcz.) Baill. Using GC-MS and chemometric resolution, Phytochem Anal, 14(1), 23-33.

[17]. Liu YM, Sheu SJ, Chiou H, Chang SH and Chen YP. A comparative study on commercial samples of ephedrae herba. Planta Medica, 1993, 59, 376-378.

[18]. Liu YM, SheuSJ. Determination of quaternary alkaloids from Coptidis Rhizoma by capillary electrophoresis, J. Chromatogr., 1992, 623(1), 196-199.

[19]. Stuppner H, Sturm S, Konwalinka G. 1992. Capillary electrophoresis analysis of oxindole alkaloids from uncaria tomentosa, J. Chromatogr. 609, 1/2, 375-380.

[20]. Liu YM, SheuSJ. Determination of coptisine, berberine and palmatine in traditional chineese Medicinal preparations by capillary electrophoresis, J. Chromatogr., 1993, 639(2),322-328.

[21]. Zhang H. 2004. Identification and determination of the major constituents in traditional Chinese medicine, Si-Wu-Tang by HPLC coupled with DAD and ESI-MS. J. Pharm.Biomed. Anal, 34,705,713.

[22]. Nyiredy S. Progress inforced flow planar chromatography, J. Chromatogr. A, 2003, 1000, 985- 999.

[23]. Roberts JE, Tyler VE. Tyler's Herbs of Choice. The Therapeutic Use of Phytomedicinals. The Haworth Press, New York.1997.

[24]. Gong F, Liang YZ, Xu QS, Chau FT, Leung AKM. Gas chromatography-Mass spectrometry \& Chemometric resolution applied to the determination of essential. 2001. 
[25]. Ylinen M , Naaranlahti T, Lapinjoki S, Huhtikangas A, Salonen ML, Simola LK, Lounasmaa M. Tropane alkaloids from Atropa elladonna part-I. capillary gas chromatographic analysis, Planta Med. 1986, 52, 2, 85-87.

[26]. Maillard MP, Wolfender JL, Hostettmann K. Use of liquid chromatography thermospray mass spectrometry in phytochemical analysis of crude plant extract, J. Chromatogr., 1993, 647, 147-154.

[27]. Mellon FA, Chapman JR, Pratt JAE. Thermospray liquid chromatography- mass spectrometry in food and agricultural research, $J$. Chromatogr. 1987, 394, 209-222.

[28]. Rajani M, Ravishankara MN, Shrivastava N, Padh H. 2001. A sensitive high performance thin layer chromatography method of estimationof diospyrin, a tumor inhibiting agent from stem bark of Diospyros Montana, J. Planar Chromatogr. 14,3.

[29]. Revilla E, Beneytez EG, Cabello F, Ortega GM, Ryan JM. Value of high performance liquid chromatographic analysis of anthocyanins in the differentiation of red grape cultivars and red wines made from them, J. Chromatogr. A, 1996, 915, 53-60.

[30]. Wolfender JL, Maillard MP, Hostettmann K. 1994. Thermospray liquid chromatography mass spectrometry in phytochemical analysis, Phytochem. Anal. 5,199.

[31]. Wolfender JL, Maillard MP, Hostettmann K. 1993. Liquid chromatographic thermospray mass spectrometric analysis of crude plant extracts containing phenolic and terpene glycosides, J. Chromatogr. 647, 183-190.

[32]. Nikam P, Kareparamban J, Jadhav A, Kadam V, Future Trends in Standardization of Herbal Drugs, Journal of Applied Pharmaceutical Science. 2012, 02(06), 38-44.

[33]. Stahl E. 1969. Thin layer chromatography, Springer verlag Berlin Heidel berg, New York, Springer international student edition.

[34]. Srivastava S, Mishra N, Genetic Markers - A Cutting-Edge Technology in Herbal Drug Research, Journal of Chemical and Pharmaceutical Research, 2009, 01(01),1-18.

[35]. Houghton P, Mukherjee P, Evaluation Of Herbal Medicinal Products, 1st ed. Pharmaceutical press; 2009, 396-421

[36]. Choudhary N, Sekhon B, An overview of advances in the standardization of herbal drugs, J Pharm Educ Res, 2011,02(02), 55-64

[37]. Kumar H, A Textbook on Biotechnology, 2nd ed. East West Press; 2001, 251-257.

[38]. Purohit, Kakarani, Saluja, Concepts, Perspectives and Techniques Of Microbial Genetic and Protein Engineering in Pharmaceutical Biotechnology, Agrobios India. 2007, 74-78.

[39]. Govil J, Singh V, Recent approaches in herbal drug standardization in Recent progress in Medicinal Plants, Standardization of Herbal/Ayurvedic formulations. 24, 25-35. 